\title{
UNIQUENESS RESULTS FOR GROUPS OF MEASURE PERSERVING TRANSFORMATIONS
}

\author{
ROBERT R. KALLMAN
}

\begin{abstract}
Let $G$ be the group of measurable, invertible, measure preserving transformations either of the unit inverval or of the line. Then $G$ has a unique topology in which it is a complete separable metric group.
\end{abstract}

1. Introduction. Let $G_{1}$ be the group of measurable, invertible, measure preserving transformations of the unit interval, and let $G_{2}$ be the group of measurable, invertible, measure preserving transformations of the line. $G_{1}$ and $G_{2}$ are complete separable metric groups in the coarse topology. For each positive integer $n \geqslant 1$, let $K_{n}$ be either $G_{1}$, or $G_{2}$, or the identity. Let $K=\Pi_{n \geqslant 1} K_{n} . K$ in a natural mianner is a complete separable metric group. The purpose of this paper is to prove the following theorem.

THEOREM 1.1. Let $H$ be a complete separable metric group, and let $\psi: H \rightarrow K$ be an algebraic isomorphism. Then $\psi$ is a topological isomorphism.

This result seems to be new even if $K$ has only one nontrivial factor. Note that Theorem 1.1 really says something about $K$, for the analogous statement for the additive group of the reals or the circle group is false. There does not seem to be any precedent for this result in the literature.

The proof of Theorem 1.1 is carried out in $\$ 2$. It is a consequence of the theory of functions with the Baire property (Kuratowski [2]). $\$ 3$ is devoted to a corollary of Theorem 1.1, namely, that there is no standard Borel group structure on the abstract group $K$ which admits a $\sigma$-finite Borel measure $\nu$ so that $\nu$ and all its translates have the same null sets (provided $K$ is not the identity). This provides an alternative proof and generalization of a result of R. J. Aumann (and independently, H. Furstenberg) [1].

One naturally might ask if there is an analogue of Theorem 1.1 if each $K_{n}$ is a group of measure preserving homeomorphisms or diffeomorphisms on a decent space which satisfies certain (hopefully) mild requirements. This is indeed the case, though the manner of proving such an analogous result is quite different in character from the proof of Theorem 1.1.

Received by the editors October 12, 1984.

1980 Mathematics Subject Classification. Primary 28D15, 28D99; Secondary 22A05.

01985 American Mathematical Society $0002-9939 / 85 \$ 1.00+\$ .25$ per page 
2. Proof of Theorem 1.1. As is usual in this subject, notions such as equality, contained in, etc., are understood to be true if they are strictly true modulo sets of Lebesgue measure zero. Time and again use is made of the fact that two measurable sets of the same positive finite measure may be interchanged by a measure preserving transformation which is the identity off their union.

Theorem 1.1 is proved gradually in a sequence of lemmas which apply to special cases. Until further notice, assume that $K$ has only one nontrivial factor $G$. If $A$ is a set, $A^{c}$ denotes its complement. For any measurable subset $A$ of the line or unit interval, let $G(A)=\left[g\right.$ in $G \mid g$ is the identity on $A^{c}$ ].

LeMma 2.1. The centralizer of $G(A)$ in $G$ is $G\left(A^{c}\right) . \psi^{-1}(G(A))$ is closed.

Proof. $G\left(A^{c}\right)$ certainly is contained in the centralizer of $G(A)$ in $G$. Conversely, suppose $g$ commutes with every element of $G(A)$. If $g \mid A$ is not the identity, then there is a measurable subset $B$ of $A$ of very small positive measure so that $g(B)$ and $B$ are disjoint. Choose an element $h$ of $G(A)$ which maps $B$ onto a subset $h(B)$ of $A$ which is disjoint from $B$ and $g(B)$, and which is the identity off of $B$ and $h(B)$. Then $g(h(B))=h(g(B))=g(B)$, which implies that $h(B)=B$. Contradiction. Hence, $g \mid A$ must be the identity. $\psi^{-1}(G(A))$ is the centralizer of $\psi^{-1}\left(G\left(A^{c}\right)\right)$ in $H$, and so $\psi^{-1}(G(A))$ is closed. This proves Lemma 2.1 .

If $A$ and $B$ are measurable sets of positive measure, let $C(A, B)=[g$ in $G \mid g(A)$ is contained in $B$ ]. The set $C(A, B)$ is a closed subset of $G$.

LEMMA 2.2. The set $C(A, B)=\left[g\right.$ in $G \mid$ every $g h_{1} g^{-1}$ commutes with every $h_{2}$, where $h_{1}$ is in $G(A)$ and $h_{2}$ is in $\left.G\left(B^{c}\right)\right]$.

Proof. Every $g h_{1} g^{-1}$ commutes with every $h_{2}$ if and only if every $g h_{1} g^{-1} \mid B^{c}$ is the identity, if and only if every $h_{1} g^{-1}\left|B^{c}=g^{-1}\right| B^{c}$, if and only if $g^{-1}\left(B^{c}\right)$ is contained in $A^{c}$, if and only if $g(A)$ is contained in $B$. This proves Lemma 2.2.

Corollary 2.3. The set $\psi^{-1}(C(A, B))$ is closed.

Proof. By Lemma $2.2, \psi^{-1}(C(A, B))=\left[h\right.$ in $H$ levery $h h_{1} h^{-1}$ commutes with every $h_{2}$, where $h_{1}$ is in $\psi^{-1}(G(A))$ and $h_{2}$ is in $\left.\psi^{-1}\left(G\left(B^{c}\right)\right)\right]$. Thus, it is now obvious that $\psi^{-1}(C(A, B))$ is closed in $H$, and Corollary 2.3 is proved.

Let $\mu$ be Lebesgue measure. Let $A$ be a measurable subset of positive finite measure, and let $B$ be a measurable set of finite measure which contains $A$, and so that $\frac{1}{2} \geqslant \mu(A) \geqslant \mu(B-A)=\delta>0$.

Lemma 2.4. The set $G\left(A^{c}\right) \cdot C(A, B)=[g$ in $G \mid \mu(A \Delta g(A)) \leqslant 2 \delta]$, and hence $G\left(A^{c}\right) \cdot C(A, B)$ is closed. $\psi^{-1}\left(G\left(A^{c}\right) \cdot C(A, B)\right)$ is an analytic subset of $H$.

Proof. Let $g_{1}$ be in $G\left(A^{c}\right)$ and $g_{2}$ in $C(A, B)$. Let $C=g_{2}(A) \cap(B-A)$ and $D=A-g_{2}(A)$. Then $\mu(C)=\mu(D), A \Delta g_{2}(A)=C \cup D$, and $\mu\left(A \Delta g_{1} g_{2}(A)\right)=$ $\mu\left(A \Delta g_{2}(A)\right)=\mu(C \cup D)=2 \mu(C) \leqslant 2 \delta$.

Conversely, let $C^{\prime}=A \cup g(A)$. Since $\mu(A \Delta g(A)) \leqslant 2 \delta, \mu\left(C^{\prime}\right) \leqslant \mu(A)+\delta$. Choose a measurable set $C$ which contains $C^{\prime}$ so that $\mu(C-A)=\delta$, and choose $g_{1}$ in $G\left(A^{c}\right)$ so that $g_{1}(B)=C$. Then $g_{1}^{-1} g(A)$ is contained in $B, g_{1}^{-1} g$ is in $C(A, B)$, 
and $g=g_{1} \cdot g_{1}^{-1} g$. This proves that $G\left(A^{c}\right) \cdot C(A, B)=[g$ in $G \mid \mu(A \Delta g(A)) \leqslant 2 \delta]$. From this it easily follows that $G\left(A^{c}\right) \cdot C(A, B)$ is closed.

$\psi^{-1}\left(G\left(A^{c}\right)\right)$ and $\psi^{-1}(C(A, B))$ are closed subsets of $H$ by Lemma 2.1 and Corollary 2.3. $\psi^{-1}\left(G\left(A^{c}\right) \cdot C(A, B)\right)$ is the continuous image of $\psi^{-1}\left(G\left(A^{c}\right)\right) \times$ $\psi^{-1}(C(A, B))$, and so is an analytic set. This proves Lemma 2.4 .

Proof of Theorem 1.1. Sets of the form [ $g$ in $G \mid \mu(A \Delta g(A))<\lambda$ ], where $A$ is a measurable set of finite positive measure, and their translates generate the topology of $G$. Hence, each open subset of $G$ is in the $\sigma$-algebra generated by the translates of the sets $G\left(A^{c}\right) \cdot C(A, B)$ of Lemma 2.4. Hence, if $B$ is a Borel subset of $G, \psi^{-1}(B)$ is in the $\sigma$-algebra generated by the analytic subsets of $H$, by Lemma 2.4 .

The remainder of the proof of Theorem 1.1 in this special case may now be carried out in standard fashion. Results from Kuratowski [2] imply that there is a residual set $H^{\prime}$ in $H$ such that $\psi \mid H^{\prime}$ is continuous. It follows that $\psi$ is actually continuous on all of $H$. To see this, let $h_{n}(n \geqslant 1)$ and $h$ be elements of $H$ so that $h_{n} \rightarrow h$. The union of $h^{-1}\left(H-H^{\prime}\right)$ and of the $h_{n}^{-1}\left(H-H^{\prime}\right)(n \geqslant 1)$ is a set of first category. Hence, there is an element $h^{\prime}$ in the complement. Then $h h^{\prime}$ is in $H^{\prime}$ and $h_{n} h^{\prime}$ is in $H^{\prime}$ $(n \geqslant 1)$. But $h_{n} h^{\prime} \rightarrow h h^{\prime}$ and so $\psi\left(h_{n} h^{\prime}\right) \rightarrow \psi\left(h h^{\prime}\right)$. Hence, $\psi\left(h_{n}\right) \rightarrow \psi(h)$. Hence, $\psi$ is continuous. Souslin's theorem implies that $\psi^{-1}: G \rightarrow H$ is a Borel mapping, and so, repeating the above argument, $\psi^{-1}$ is continuous. Hence, $\psi$ is a topological isomorphism, and Theorem 1.1 is proved in this case.

To prove Theorem 1.1 in general, fix a positive integer $n \geqslant 1$, let $U$ be open in $K_{n}$, let $K_{n}^{\prime}=\prod_{m \geqslant 1, m \neq n} K_{m}, H_{n}=\psi^{-1}\left(K_{n}\right)$, and $H_{n}^{\prime}=\psi^{-1}\left(K_{n}^{\prime}\right) . H_{n}$ and $H_{n}^{\prime}$ are closed in $H$, for each is the centralizer of the other. $H_{n}$ and $H_{n}^{\prime}$ intersect in the identity, and $H=H_{n} \cdot H_{n}^{\prime}$ as an abstract group. Hence, the argument given in the previous paragraph, for instance, shows that $H=H_{n} \times H_{n}^{\prime}$ is a topological group. By the special case already proved, $\psi^{-1}\left(U \times K_{n}^{\prime}\right)=\psi^{-1}(U) \times H_{n}^{\prime}$ is an open subset of $H$. Since the topology of $K$ is generated by sets of the form $U \times K_{n}^{\prime}, \psi$ is continuous. The proof of Theorem 1.1 may now be finished off by the same argument used in the previous paragraph.

3. Nonexistence of quasi-invariant measures. See Mackey [3] for the background on standard Borel groups used in the following corollary.

COROLlaRY 3.1. Suppose the $K$ of Theorem 1.1 has at least one nontrivial factor. There is no analytic Borel structure $\mathscr{B}$ on $K$, with respect to which $K$ is an analytic Borel group, and which admits a $\sigma$-finite Borel measure $\nu$ on $\mathscr{B}$, all of whose left translates under $K$ have the same null sets. In particular, $K$ cannot be given the structure of $a$ locally compact group with a countable basis for its topology.

Note that there is no a priori reason to believe that $\mathscr{B}$ has any connection with any topological group structure on $K$. A weaker result has been proved by R. J. Aumann (and independently, H. Furstenberg) [1] by quite different methods.

The following simple lemma is needed first.

LEMMA 3.2. If $K$ has a nontrivial factor, then $K$ is not locally compact. 
Proof. It suffices to prove this for $G_{1}$. For each integer $n$, let $C_{n}=\{0,1\}$, viewed as a discrete topological space with a measure which assigns measure $\frac{1}{2}$ to each point. Let $C=\Pi_{n} C_{n}$, a compact metric space and a measure space with the product measure. The infinite symmetric group $S_{\infty}$ acts on $C$ by permuting coordinates and preserves the product measure. Check that the natural mapping of $S_{\infty}$ into the group of measure preserving transformations of $C$ is in fact an embedding. Since $C$ with the product measure is the same measure theoretically as the unit interval with Lebesgue measure, the proof of the lemma is finished once one notes that $S_{\infty}$ is not locally compact, for any neighborhood of the identity in $S_{\infty}$ contains a subgroup which has unbounded orbits on the integers.

Proof of Corollary 3.1. Suppose that there is some analytic Borel structure $\mathscr{B}$ on $K$, with respect to which $K$ is an analytic Borel group, and which admits a $\sigma$-finite Borel measure $\nu$ on $\mathscr{B}$, all of whose left translates under $K$ have the same null sets. Theorem 7.1 of Mackey [3] implies that $K$ can be made into a locally compact group with a countable basis for its topology, and so that $\mathscr{B}$ is the collection of Borel sets with respect to this topology. But Theorem 1.1 implies that $K$ has a unique topology in which it is a complete separable metric group. Lemma 3.2 implies that this topology is not locally compact. Contradiction. This proves Corollary 3.1 .

\section{REFERENCES}

1. R. J. Aumann, Random measure preserving transformations, Proc. Fifth Berkeley Symposium on Mathematical Statistics and Probability, Vol. 2, Part 2, Univ. of California Press, Berekeley, Calif., 1967, pp. 321-326.

2. K. Kuratowski, Topology. Vol. I, Academic Press, New York, 1966.

3. G. W. Mackey, Borel structures in groups and their duals, Trans. Amer. Math. Soc. 85 (1957), 134-165.

Department of Mathematics, North Texas State University, Denton, Texas 76203 